\title{
Effective Construction Process Monitoring and Control through a Collaborative Cyber-Physical Approach
}

\author{
Yaseen Srewil and Raimar J. Scherer \\ Institute of Construction Informatics, Technische Universität Dresden, \\ Dresden, Germany \\ \{Yaseen.Srewil, Raimar.Scherer\} atu-dresden.de
}

\begin{abstract}
The objectives of this research are: monitoring of project progress during execution, assessment project health on demand, to identify and make timely recommendations for corrective action in response to anticipated schedule delays. Implementation of this information in flexible process modeling approaches, like process configuration method enhances an alternative process planning. The paper proposes a solution in collaboration with cyber-physical system CPS based on RFID technology to minimize manual inputs and enhance data acquisition. Furthermore, it enables planers anticipating and identifying schedule delays and exceptions early or even before they happen. Analysis of the real-time data collected on site and actual vs. scheduled deviation will be transformed into a meaningful classification. This paper presents a comprehensive solution to integrate the outcomes of analysis real-time data into a knowledge base for updating the entire progress, handling exceptions and supporting efficient process alternative modeling.
\end{abstract}

Keywords: RFID, CPS, Process Monitoring and Control.

\section{Introduction}

The efficient process planning in construction project is still confronted with insufficient information about the current process and necessary changes in the process flow realized in project execution. In many field practices, the planners still rely on manual process methods to collect information during project execution. Thus, the information of actual construction process is incomplete, error-prone and not available on time. Indeed, the information problem has $27 \%$ of disturbance-causes in construction project [1]. Furthermore, the missing integration of the information in a flexible process modeling hinders an alternative process planning and causes process delays. To ensure an effective continuous information flow, communication, and collaboration among all involved actors in project execution, a cyber-physical approach is proposed. The suggested solution enables coupling the real world with the virtual one together to produce an environment where the physical and digital objects interact in real-time. Radio Frequency Identification (RFID) technology is used as coupling technology for identification of construction site resources. 
The objectives of this research are: (1) monitoring of project progress during execution, (2) assessment project "health" on demand, to identify and make timely "recommendations" for corrective action in response to anticipated schedule delays and (3) implementation of this information in flexible process modeling approaches, like process configuration method enhances an alternative process planning.

At this, the paper proposes (1) a mechanism to minimize manual input by enhancing data acquisition and collaborative interaction of virtual model with physical environment, (2) an approach to identify schedule delays (exceptions) early or even before they happen and (3) monitoring project "health" and analyzing realtime data. Thereby, the focus will lay on analysis of collected data, determination of actual-schedule date deviations and suggest a classification approach of classes in comparison with project thresholds, priority of an activity (belongs to the critical path of the project) and the floating time of an activity.

Finally, a comprehensive solution to integrate outcomes of analyzed data into a knowledge base will be presented for updating the entire process progress, handling exceptions execution and supporting efficient modeling of process alternative.

This work discusses primary the prefabricated components of Fiber Reinforced Polymer that can be used in modern bridge construction and their lifecycle (shipping and on site assembly). Since, these components have a high initial material cost which makes the overall project cost very sensitive to errors. Therefore, this work is driven by the "need" for accurate records of components and their status on construction site. The presented research is part of the ongoing FP7 European Project Trans-IND (New Industrialised Construction Process for transport infrastructures based on polymer composite components).

\section{Background}

Recently, several researches have been investigated the integration of physical construction components and their virtual models using RFID technology. However, the early research in construction industry was focused on RFID applications for concrete handling, cost coding, material control and tool tracking onsite [2]. Later, a new tracking architecture based on an embedded system for tracking construction asset by combining RFID and ultrasound signal is introduced [3]. Product data management (PDM) systems and near field communication (NFC) technology is developed in framework of FORBAU project to provide the construction project progress data to all involved actors [4]. Studies were made to measure the construction project progress by proposing a control model based on automated data collection form work-site [5] and a framework for automated monitoring system for steel structure construction [6]. Another approach suggested a solution according to knowledge management system comprising three components: RFID data component, knowledge base, and optimization component. Thereby, the core of the knowledge base is formed by ontology, which describes in a formal matter the contents and relations relevant for modeling on-site processes [7]. One of the current research work proposed a solution based on Real-Time Location Sensing (RTLS) system to enable a Cyber-Physical System (CPS) for information exchange [8]. Although this approach 
has a promise results; the expense of such a solution hinders a wide application in construction industry. Finally, the potential integration of RFID technology is evident by the Institute of Construction Informatics to control the dynamic construction process throughout coupling of flexible process models and the physical construction using cheaper passive RFID tag [9].

\section{Cyber-Physical System (CPS) Approach}

From a constructional point of view, CPS can be defined as a tight coupling between virtual models and physical construction to enable data bidirectional coordination [8]. Basically, the CPS architecture (as in fig. 1) encompasses the virtual models, physical entities and interfaces in both directions (cyber to physical interface and v.v.) based on data acquisition technologies like RFID. CPS enables bidirectional coordination between physical construction components and their virtual model in real-time. Hence, CPS is not only a part of the passive identification process but also a part of the active response and collaborative procedures as it is closely integrated with the control system. It endorses the challenge of ensuring accurate and timely update of information during construction process.

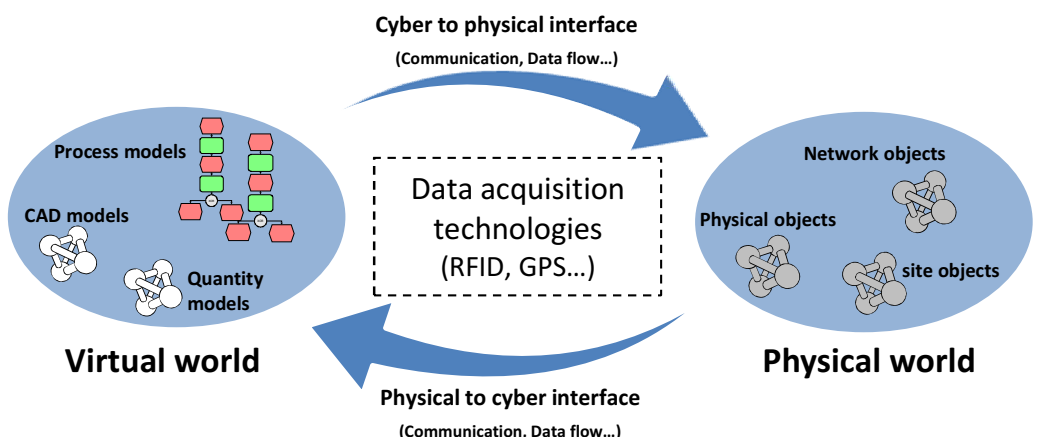

Fig. 1. Principle CPS architecture: interaction between virtual and physical world problem via data acquisition technologies (like RFID system, GPS, Sensors...)

\subsection{Data Acquisition Technologies}

The process of collecting data can be automated by emerging Automatic Data Capturing (ADC) technologies (RFID, GPS, Barcode, Laser scanning, etc.). It represents the technical part of CPS. In construction industry, these modern technologies are still under development, and the feasible applications are available only in few prototypes. Since RFID has relative advantages over other technologies, it is used to generate and aggregate information about real world objects (i.e. Products, equipment, shipments and personal information). RFID can facilitate progress measurement through the set of captured data and monitoring of project's status.

The RFID tag can be classified mainly as passive, active or semi-active. The passive tags receive power to transmit data from the reader. They have a cheap price, 
small data storage capacity and short read range. The active tags use a built-in battery to transmit data. Typically, they are expensive, have high data storage capacity, and a long reading range [10]. Thanks to their excellent features for the identification of static or even moving products, machines, and crew in harsh environment, Ultra High Frequency (UHF) RFID systems can be used in this proposed system.

\subsection{Site Organization to Leverage Collaborative Data Acquisition Technology}

To allow a continuous monitoring of all relevant components and resources throughout all construction phases, a methodology is required for detecting these physical objects, their positions, and their states undisturbed, automated and in realtime. This methodology bases on organizing the construction site to leverage collaborative RFID technology. Figure 1 illustrated approach considers three characteristic layers of job site: (1) Event layer representing where the construction processes take place, (2) Network layer for the mapping of events from the event layer to network configurations to make them tractable and (3) construction site layout layer; it links the network devices to their corresponding physical locations.

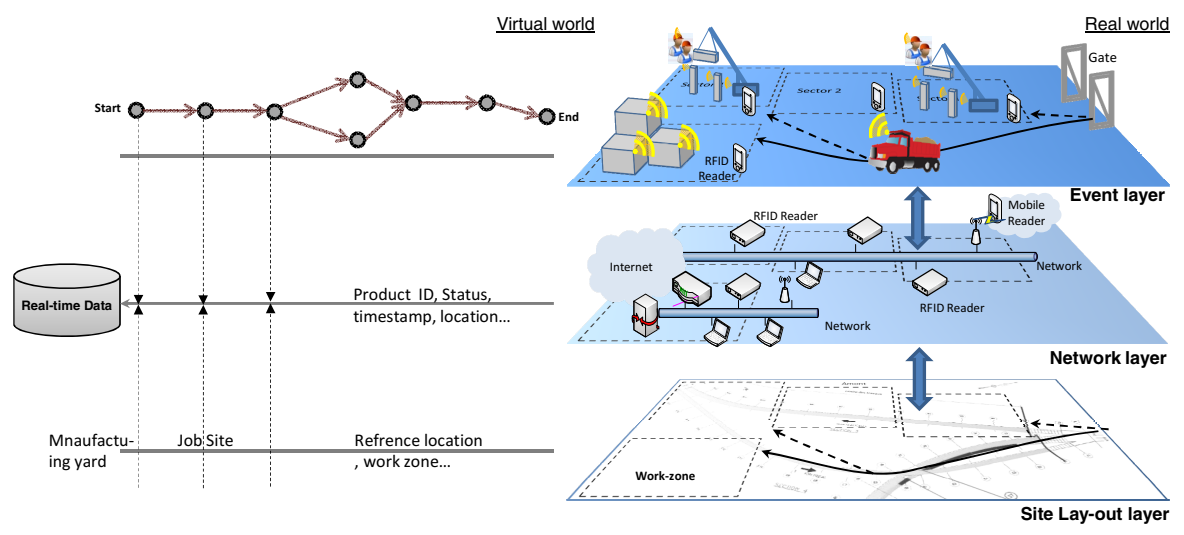

Fig. 2. A concept system for embedded UHF RFID technology based on RFID reader

Breaking down the construction site into work zone enables predefined entrances of the construction site, stores and lay down areas, and constructed facility places. At this, the components status can be easily updated at the job site gate, store or lay down areas, cranes at constructed facility site, etc. These places are mounted with RFID reader (stationary and/or mobile). Accordingly, the data (Product ID, timestamp, product status, location, etc.) can be gathered automatically at each process level, or semi-automatically by the foremen, who can also add their comments. Therefore, the suggested system keeps the process information continuously updated. Lastly, the collected real-time data will be handled in a knowledge base. This site origination solution leverages collaborative data acquisition technology and can dramatically minimize the time to update the state of each object towards automated data collection on construction site. 


\section{Real-Time Monitoring and Progress Determination}

Real-time monitoring is a continuous process that takes place during the whole lifecycle of the construction phases. It links the field where physical objects are processed on the proposed CPS to the virtual one via RFID, which provides a stream line of data (IDs, time, location...) for all resources on site; therefore a real-time status update is on hand. Several research works were made to manage and transform raw data into meaningful events that can be understood by the applications. The realtime monitoring process requires a harmonization of construction process and collaboration of CPS which can be summarized in following sub-processes:

- Managing RFID data: Generally, the major steps for managing RFID data are data capturing, data filtering, and event processing and integration onto an application which was discussed in detail in previous work (cf. [9], [11]).

- Identifying related construction process from the virtual part of CPS: The entire construction project is described by a set of construction processes or activities depending on the level of description; each process needs to be accomplished within a defined period of time. These processes can be extracted according to Work Breakdown Structure (WBS) of the project. To identify the processes affected by a triggered event, the updated resources within a certain process window containing the current processes and the next direct successors will be detected. Then, the formulated relationships between processes and resources allow the identification of the corresponding processes.

- Deriving process progress: To derive the current progress of the identified processes. A few indictors corresponding to RFID application scenarios are applied. These indicators depend on the process duration, process dependencies and the required resources. Thereby, the underlying metric and the available information of the resources influence how detailed the process progress can be specified. On the next level of detail, the exact start and end time can be indicated. A further detailed specification provides information about the certain process state during its execution.

Key Performance Indicators (KPIs) are used to measure the progress of the assembly process of prefabricated components on site and. KPIs commonly aim to evaluate the success and the performance of a company. In this approach, KPIs are considered to determine the progress of ongoing assembly processes. Two indicators are discussed:

- The components availability: Each element (prefabricated elements, palettes...) is associated with a RFID tag. While elements move through different phases (logistic and/or on-site) the corresponding RFID tags are tracked by RFID readers. The retrieved information consists of Tag-ID, the Reader-ID and the current reading Timestamp as well as reader's position. This information will be stored and regularly updated into a knowledge base. The querying of an Element -ID and its last location in well predefined construction site (Fig. 2) can concern the availability of this element and its current status [9].

- Process time: It is an important indicator for process progress tracking. Estimating the process time is a complex task. However, the approximate process time can be estimated by identifying the start and end dates for each activity. The events dates are determined when the objects changed their statues. These dates are considered 
as start and end of the related activity. For instance, beam assembly process starts as soon as the beam state changed from beam-stored into beam- in assembly and it ended as its status changed into beam-mounted. The aggregated dates are used for estimating the whole process time and hence the progress of these processes.

\section{$5 \quad$ Real-Time Data Analysis and Classification}

This step aims to detect process deviations and achieve a continuous comparison of actual with planned performance. Here, the accomplished detection of deviations is for both prediction and actual values at different levels of detail (process or even activity). The process in this case can be defined as a set of activities. The comparison comprises the resource (as build, as planned) and the date variations of current process. Based on deviation values, the outputs can be categorized into three classes according to the project thresholds, the activity priority (belongs to the critical path of the project), and the floating time of an activity (Fig. 3). These classes are:

- Class1: early, on schedule "or even very close to" $\rightarrow$ no or accepted risk detected.

- Class2: nearly on schedule $\rightarrow$ risk or potential risk detected.

- Class3: late (i.e. activity on critical path of project) $\rightarrow$ problem detected.

Depending on these outputs the process states will be derived, detecting delays in project execution and planning for alternative which is discussed in the next section.

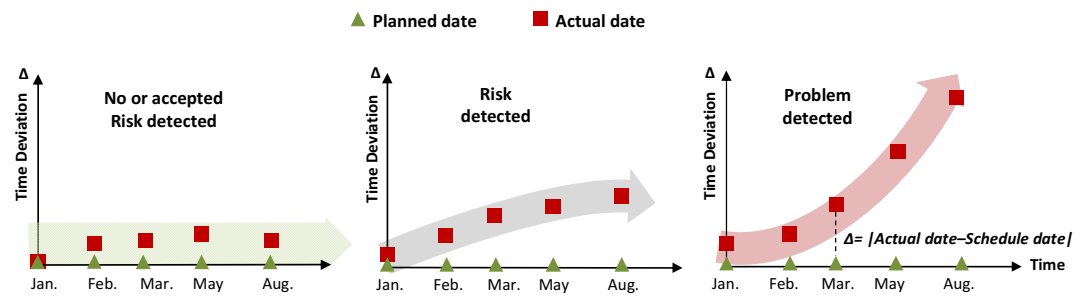

Fig. 3. Outputs of analysis real-time monitoring data of project activities "actual vs. schedule"

\section{Construction Process Monitoring and Control}

Real-time monitoring of construction processes is necessary to anticipate and identify schedule delays and exceptions at the early stage and even before they happen. Nevertheless, the analysis of these data facilitates the assessment of project health and to make timely recommendations for corrective action in response to these exceptions. Because of construction project nature, some exceptions (risks) can be realized early at the planning phase. However, the realism of the risk assessment increased as project proceeds (i.e. considering a new risk and omitting others). This work proposes a comprehensive solution (Fig. 4) for construction process monitoring and control in real-time and a mechanism to add corrective action during execution in collaboration with well-known flexible process modeling method. This solution comprises the following three main components: 
- The real-time monitoring represents data aggregation and analysis that was classified above. These results can be derived in more specific identification and assessment steps (beyond as-build/as planned assessment) (cf. [12]).

- The second part is a suggested mechanism to add suitable treatments and recommended actions. These treatments can be an Ad-hoc solution, or configurable fragments (preplanned treatment) added to the process model as proactive treatment and to configure such configurable fragments as reactive treatment based on continual monitoring of process execution [10]. Hence, the treatment is a standardized process description that offers enough flexibility to be used in a different context. And the configurable fragments in the course of the process model express the uncertain parts of the process. Recent research work proposes to use templates that are a type of configurable fragments within the process models. These templates are generic and supposed to provide the flexibility needed in the process model [13], [14]. The real-time monitoring of ongoing activities can support the configuration of such templates by selecting the most suitable fragment for each variation point.

- Finally, all alterations are documented, the process models are updated to the project knowledge base and the monitoring cycle is resumed. Knowledge base roles are to manage the entire process model, formulate dependencies in a logicbased manner and to derive additional information required for filtering events, identifying risk types, configuring process fragments.

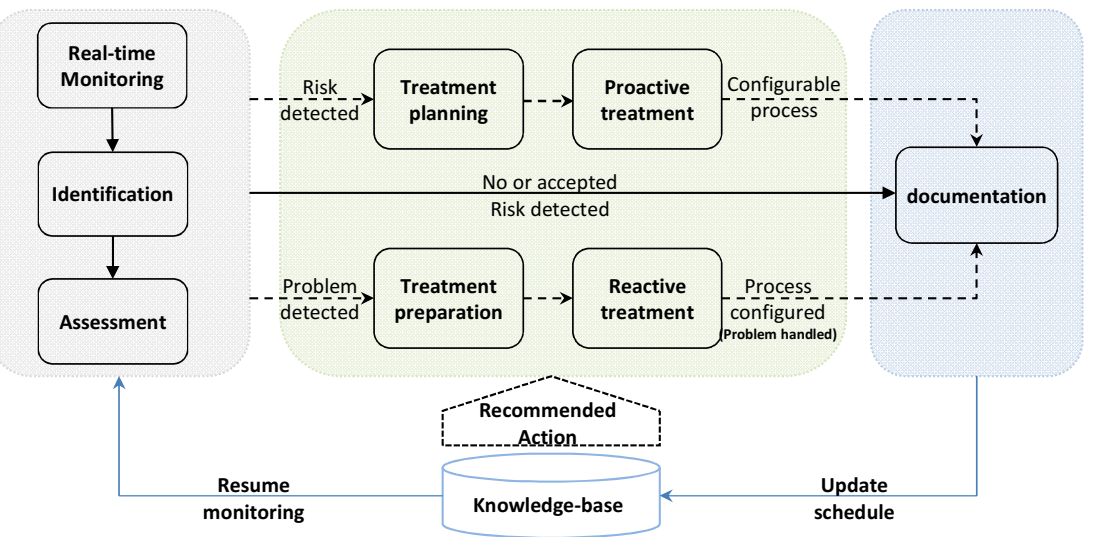

Fig. 4. Comprehensive approach based CPS for real-time monitoring control changes

\section{Conclusions}

The paper showed significant opportunities of using the CPS based RFID. It enhances the real-time construction process monitoring and aids an early decision making and process control. Moreover, the proposed site organization to leverage collaborative RFID system can minimize the manual inputs of data and lead to automation of data acquisition. The analysis of real-time data collected on site can give a clear view of project progress. Furthermore, it facilitates the transform of these data into 
meaningful classifications that can be understood by the applications. The direct application of this classification is to control the construction process in real time. Hence, the comprehensive real-time monitoring and control solution promises a flexible modeling method. That method is based on a mechanism to configure the recommended actions. Thereby, configurable process templates are used to respond to the frequently changes in construction site conditions.

Acknowledgments. This research is partially funded by EU-Project (FP7 no. 229142) Trans-IND. This support is gratefully acknowledged.

\section{References}

1. Gehbauer, F., Zülch, G., Ott, M., Börkircher, M.: Simulation-based analysis of disturbances in construction operations. In: Proceedings of the International Group for Lean Construction 15th Conference (IGLC-15), Michigan (2007)

2. Goodrum, P.M., McLaren, M.A., Durfee, A.: The application of active radio frequency identification technology for tool tracking on construction job sites. Autom. Constr. 15, 292-302 (2006)

3. Won-Suk, J., Skibniewski, M.J.: Embedded system for construction asset tracking combining Radio and Ultrasound signals. In J. Comput. Civil Eng. 23, 221-229 (2009)

4. Klaubert, C., Schorr, M., Günthner, W.: Real time construction progress control using NFC. In: Proceedings of the Systech 2010, Ciudad Real, Spain (2010)

5. El-Omari, S., Moselhi, O.: Integrating automated data acquisition technologies for progress reporting of construction projects. In: Proc. of 26th ISARC, Austin, TX (2009)

6. Azimi, R., Lee, S., AbouRizk, S., Alvanchi, A.: A Framework for Automated and Integrated Project Monitoring and Control System for Steel Fabrication Projects. Autom. Constr. 20(1), 88-97 (2011)

7. Kadolsky, M., Srewil, Y., Scherer, R.J.: Knowledge-based on site process optimization using RFID technology. In: Proceedings of 28th CIB W78 and 6th CIB W102 2011 International Conference. Biot - Sophia Antipolis, France (2011)

8. Akanmu, A., Anumba, C., Messner, J.: An RTLS-Based Approach to Cyber-Physical Systems Integration in Design and Construction. International Journal of Distributed Sensor Networks 2012, Article ID 596845, 11 (2012)

9. Srewil, Y., Kadolsky, M., Scherer, R.J.: Integrating RFID Technology in Dynamic Construction Process Planning. In: Proc. Joint 29th CIB W78, Beirut, Lebanon (2012)

10. Erabuild: RFID in construction. ERABUILD final report (2006)

11. Li, Z., Chu, C.-H., Yao, W.: SIP-RLTS: An RFID Location Tracking System Based on SIP. In: 2008 IEEE International Conference on RFID, pp. 173-182 (2008)

12. PMBOK Guide: A guide to the project management body of knowledge. Project Management Institute (PMI), Pennsylvania, USA (2004)

13. Sharmak, W.: Dynamic Network planning in construction projects using configurable reference process models. PhD thesis: Berichte des Instituts für Bauinformatik, Heft 9, Dresden, Germany (2011) ISBN 978-3-86780-228-4

14. Scherer, R., Sharmak, W.: Process risk management using configurable process models. In: Camarinha-Matos, L.M., Pereira-Klen, A., Afsarmanesh, H. (eds.) PRO-VE 2011. IFIP AICT, vol. 362, pp. 341-348. Springer, Heidelberg (2011) 